\title{
A Volumetric Optimization of a Low-Pass Filter
}

\author{
Mark Gerber, Jan Abraham Ferreira, Ivan W. Hofsajer, Member, IEEE, and Norbert Seliger
}

\begin{abstract}
The low-pass filter (LPF) is a fundamental component of many power electronic systems, for example, the output filters of dc-to-dc converters. The volume of the two components that constitute the LPF contribute a majority of the final system's volume, thus affecting the overall power density of the system. To be able to reach the high power densities that are required nowadays, the packaging of these components needs to be addressed. A new packaging approach is considered where the main criteria is to increase the packaging density of the LPF. The filter is considered volumetrically, and the minimum possible volume is derived. The construction and losses of such a filter are also discussed.
\end{abstract}

Index Terms-Energy density, high-density packaging, loss distribution, low-pass filter, shield, volumetric optimization.

\section{INTRODUCTION}

$\mathbf{T}$ HE low-pass filter (LPF) is present in many of today's power processing systems. An example would be the output filter of a dc-to-dc converter. Such an LPF is illustrated in Fig. 1. The filter is normally realized using two discrete components that are each packaged and manufactured separately without any consideration for the other. The two components are then interconnected, normally using methods such as printed circuit boards or busbar structures. The filter, consisting of the two bulky components, usually contributes significantly to the overall volume of the system in question. With the current trend in power electronics to increase the power density [1], [3], it becomes vital to account for and eliminate any unused volume. The typical implementation of the LPF, shown in Fig. 1, shows graphically how much volume can go by unutilized. This wasted volume, if used, can help to improve the power density of the system significantly, resulting in smaller systems. On the other hand, the advantage of constructing the filter as in Fig. 1 is the ease with which the filter can be cooled. There is a large surface area present that can be used for cooling purposes. By making any changes to the construction of the LPF, such as increasing the package density, the thermal issues of the filter must be kept in mind.

Paper IPCSD 02-043, presented at the 2001 Industry Applications Society Annual Meeting, Chicago, IL, September 30-October 5, and approved for publication in the IEEE TRANSACTIONS ON INDUSTRY APPLICATIONS by the Power Electronics Devices and Components Committee of the IEEE Industry Applications Society. Manuscript submitted for review April 1, 2002 and released for publication July 16, 2002.

M. Gerber and J. A. Ferreira are with the Faculty of Electrical Engineering, Power Electronics and Electrical Machines, ITS Faculty, Delft University of Technology, 2600GA Delft, The Netherlands (e-mail: M.Gerber@ITS.TUDelft.nl; J.A.Ferreira@ITS.TUDelft.nl).

I. W. Hofsajer is with the Industrial Electronics Technology Research Group, Rand Afrikaans University, Johannesburg 2006, South Africa (e-mail: iwh@ing.rau.ac.za).

N. Seliger is with Corporate Technology, Siemens AG, D-81730 Munich, Germany (e-mail: Norbert.Seliger@mchp.siemens.de).

Publisher Item Identifier 10.1109/TIA.2002.802996.

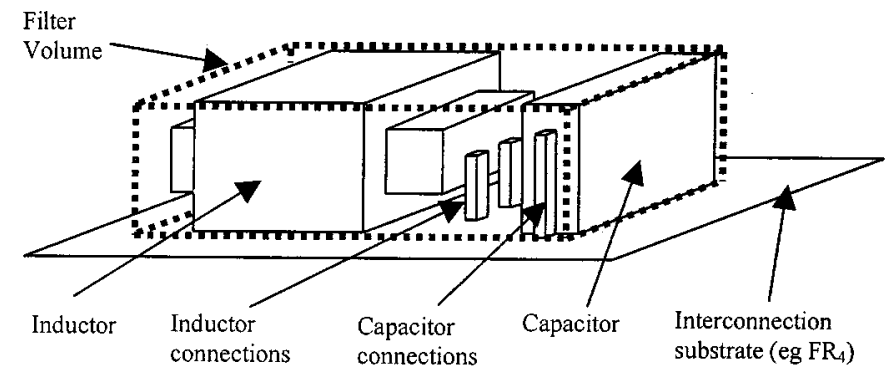

Fig. 1. Typical implementation of an LPF.

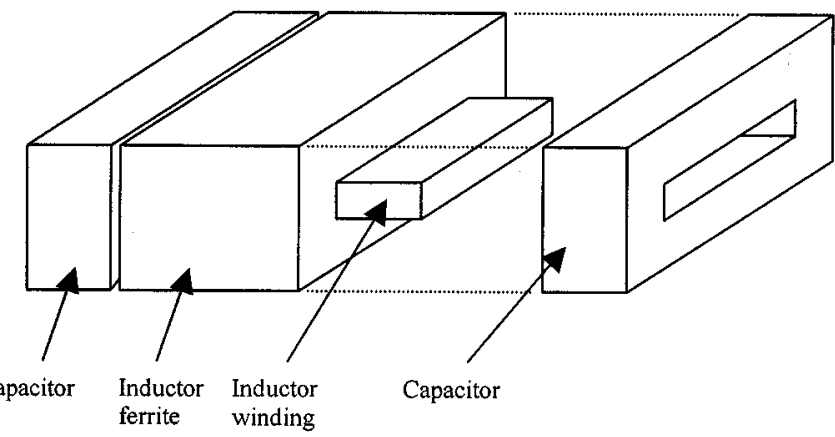

Fig. 2. Proposed LPF structure.

In this paper, the physical construction of the LPF is considered from a volume utilization point of view.

A different construction method to realize the LPF is presented and investigated. Some of the practical issues relating to the construction and interconnections within the LPF structure are explored. The structure is further investigated using a finite-element package to investigate and gain insight into the magnetic field distribution within the structure. This will inevitably lead to a better understanding of the losses within the structure.

The total volume of the filter is then optimized against the energy to be stored within the filter. Finally, a case study is considered where the LPF structure is built and investigated.

\section{INCREASING THE VOLUME USAGE}

In order to achieve a package density higher than what is currently achieved, the LPF needs to be packaged in a different manner [5]. Under normal construction conditions, the inductor and capacitor are constructed independently and without any consideration for each other. To achieve the desired package density, the two components geometries need to be changed to complement each other in such away so that they utilize the available volume and still meet the electrical and electromagnetic requirements. Fig. 2 presents such a geometry.

In Fig. 2, the capacitor has been reduced to two equal capacitors that will ultimately be reconnected in parallel. The capac- 


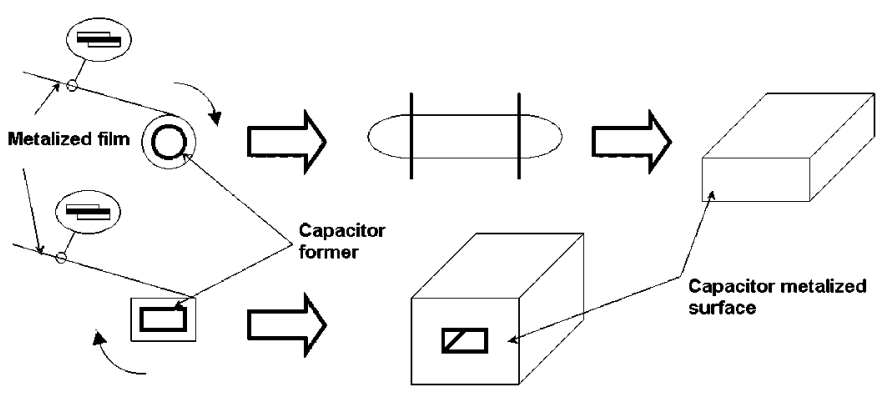

Fig. 3. Film capacitor manufacturing process.

itors are selected as the components that will undergo the constructional changes due to the higher degree of freedom they offer as compared to the inductor. The capacitors have been reshaped to complement the inductors shape and are reshaped into "square donuts" so that they fit over the windings protruding from the sides of the inductor. The inductor is implemented with standard E-cores, either steel or ferrite, and a winding construction on its bobbin.

The result is a structure that can still meet the electrical and electromagnetic requirements of the LPF while maximizing the use of the volume.

The structure, as shown in Fig. 2, raises many questions that need to be answered before the structure can be realized. The structure has the advantage of a higher package density but presents difficulties concerning the interconnections, as well as the losses within the structure. In the following sections, some suggestions are presented that can be used to overcome some of these problems.

\section{IMPLEMENTATION ISSUES}

The realization of the structure shown in Fig. 2 will be directly connected to the construction technologies that are available. In this section, some construction methods are considered based on different technologies. These will include different ways of realizing the capacitance as well as interconnections within the structure.

\section{A. Structure Realization}

1) Foil-Based Capacitor: As mentioned previously, the LPF structure consists of an inductor structure with two custom-shaped capacitors. One possible way to realize the required capacitor shape is to take advantage of metalized film capacitors [4]. The metalized film capacitors that offer the best performance for general power electronic applications are the the polypropylene-based film capacitors [4]. The polypropylene-based capacitors offer a good compromise between dielectric losses, frequency response, and stability at high operating temperatures $\left(85^{\circ} \mathrm{C}-105{ }^{\circ} \mathrm{C}\right)$. Fig. 3 shows a simplified manufacturing process for the section of the capacitor.

The standard manufacturing process, illustrated in the top of Fig. 3, begins with a dielectric foil that has been metalized. Two metalized foils are rolled together onto a former until the required number of layers is reached. The former is then removed from the rolled-up foil and the metalized foil is squashed. The result is a mass that consists of many layers of parallel con-

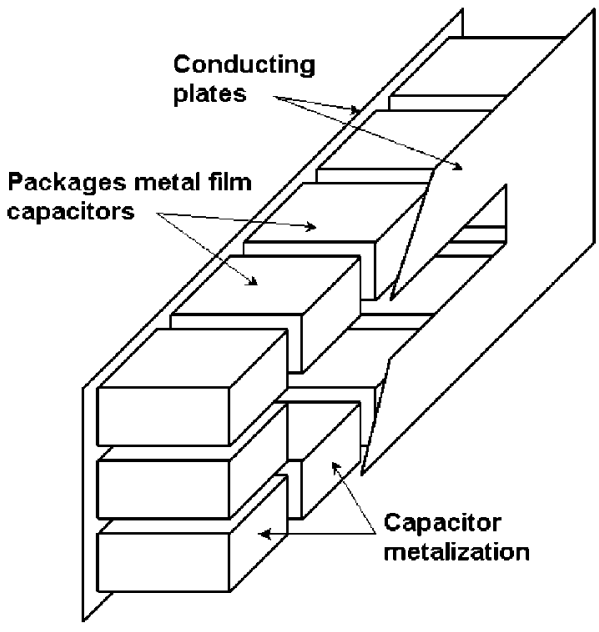

Fig. 4. Realizing the capacitor with individual metal film capacitors (half of the top plate has been removed for illustrative purposes).

ductors separated by a dielectric. At the side of the mass, the bulges are removed, reducing the mass to a rectangular shape. At each side of the rectangular mass, one of the two conductors is exposed due to the conductors position on the foil. Electrical connection is established by metalizing the exposed conductors on each side of the rectangular mass. To complete the capacitor structure, the leads are attached and it is placed in a casing.

Based on this manufacturing process, it is possible to produce the capacitor structure required to realize the LPF structure.

The capacitor structure can be achieved by replacing the initial foil former with one of the required form and dimensions. An advantage of this method would be that there would be no bulging edges that need to be removed once the capacitor has been wound. Finally, there is also no need for the former to be removed. Once the sides of the structure have been metalized, the capacitor structure is completed. However, it should be remembered that there could be electric field enhancement effects in the tight corners of the new capacitor structure that must be taken into account in the capacitor design. The alternative manufacturing process is illustrated in the lower part of Fig. 3. Manufacturing a customized metalized capacitor would provide the optimal solution, but this can be costly for a small number of capacitors. As an alternative to the customized foil capacitor, another capacitor structure is also considered.

2) Lumped-Capacitor Realization: The capacitor structure in Fig. 4 consists of several metal film capacitors that have been placed between two conducting plates. The capacitors are positioned in such a way so that the metalized sides are in contact with the conducting plates. Electrical connection between the metalized surfaces of the capacitors and the conductors can be achieved with a soldering process. This capacitor structure offers a simple solution to achieve the custom-shaped capacitor required for the LPF structure.

\section{B. Interconnections}

In this section, only three possible interconnection methods are considered. The first method makes use of standard PC board material, and the second looks at using flexible polymer substrate. The latter considers a special plastic bobbin that has the interconnections implemented within a single bobbin. 


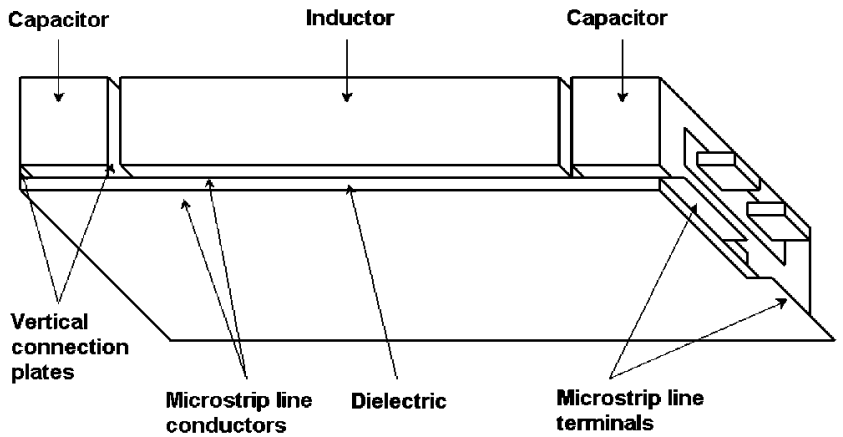

Fig. 5. LPF structure interconnections implemented with a microstrip line.

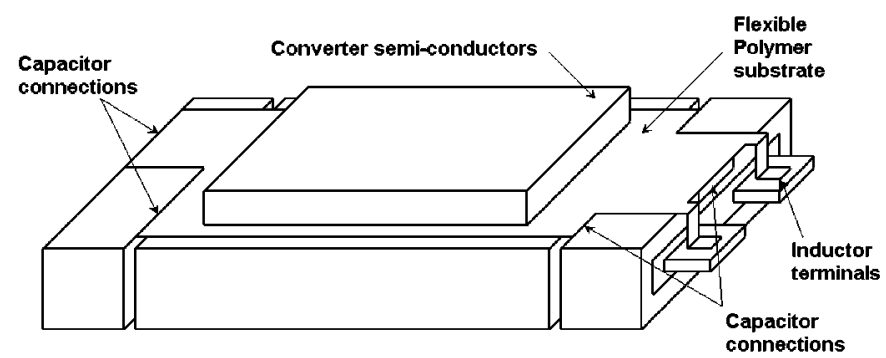

Fig. 6. Interconnections are realized with a flexible printed circuit.

1) Microstrip Line Interconnection: The microstrip transmission line is a widely used method of interconnection [2]. The microstrip line consists of a planar conductor placed above the return path separated by a dielectric material. This type of interconnection is relatively easy to implement using printed circuit board technology.

Fig. 5 shows the LPF with the interconnections between the capacitors and inductor implemented with a microstrip line. The microstrip line is planar in nature and can be sized to fit very close to the LPF structure. In doing this, the volume of the LPF structure is not increased.

2) Flexible Polymer Substrate: Electric circuits based on flexible polymer substrates offer a unique advantage to the packaging engineer. The polymer substrate can be considered to be a piece of paper that can be bent and folded into any conceivable shape. This makes the third dimension easily assessable to reduce the complexity of interconnecting dense structures. The flex technology uses industry-standard printed circuit board and/or thick film process [6] to offer a low-cost high-performance solution to the interconnection problem.

Fig. 6 shows a possible interconnection configuration that takes advantage of the high flexibility that the polymer substrate has to offer. The substrate can be bent over the side of the inductor, and contact with the capacitor conducting plates can be achieved. The connection to the inductor can be made in a similar way.

A further possibility is to include the active components on the substrate as well. Thus, it will be possible to build the complete converter on top of the LPF structure. The functionality of the two capacitors can be divided. One of the two capacitors can be used as a bus capacitor, while the second one is used for the filtering. This can allow the complete converter to be constructed in a very dense manner.

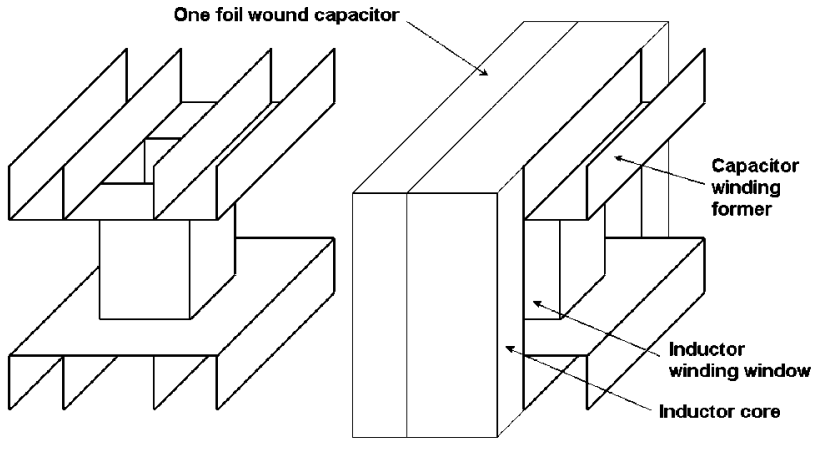

(a)

(b)

Fig. 7. (a) Cstom bobbin. (b) Custom bobbin with the inductor core and a single capacitor.

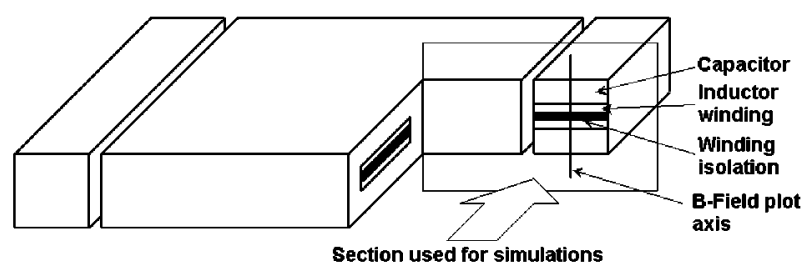

Fig. 8. Section of the LPF structure used in the simulations.

3) Customized Bobbin: In the two interconnection possibilities discussed thus far, the manufacturing process of the capacitor structure was not relevant. In other words, the methods presented required the capacitor structure to be of the correct shape and it did not matter if the capacitor were implemented with a foil-based capacitor or with several individual capacitors.

The customized bobbin, illustrated in Fig. 7, requires the capacitor to be implemented with a foil winding. The bobbin presents a method to accommodate both the inductor windings and capacitor foil windings on a single structure. The figure shows how the inductor core and the capacitor are placed on the bobbin (only one of the two capacitor structures is shown). The inductor core fits on the bobbin as per usual. To accommodate the capacitor foil winding, extra protrusions are included on the bobbin. To realize the structure, the inductor winding must be placed on the bobbin first. The figure does not show the inductor windings. Once the inductor winding is in place, the foil windings for the capacitors are then included. The procedure will be similar to that shown in Fig. 3. Once the foil has been wound, the inductor core is included, completing the structure. The interconnections within the structure can be implemented with any of the previous methods discussed.

\section{Structure Losses}

In the LPF structure, the inductor and capacitors are in very close approximation to each other. The magnetic fields associated with the inductor interacts with the capacitors, resulting in increased losses. These losses are caused primarily by eddy currents that are induced in the capacitors due to the changing magnetic field of the inductor [7].

To investigate the magnetic field distribution, a finite-element package is used. An arbitrary case study is considered in the simulations. Fig. 8 shows the section that is used in the simulations. 


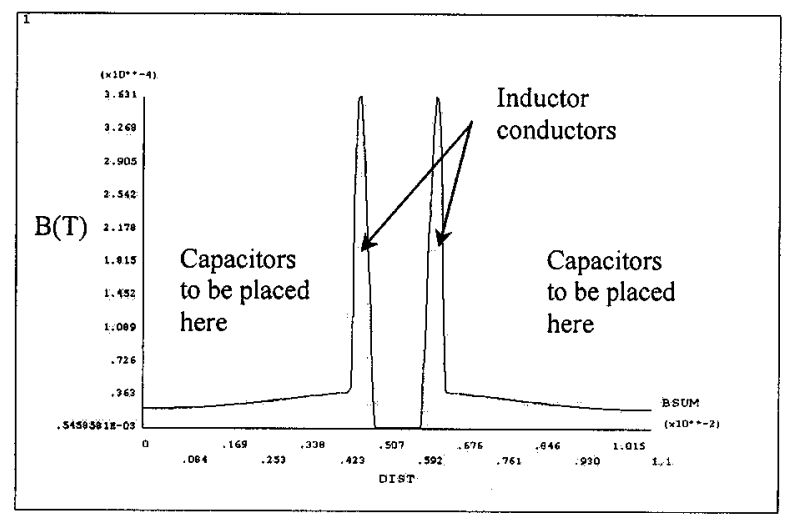

Fig. 9. Magnetic field distribution.

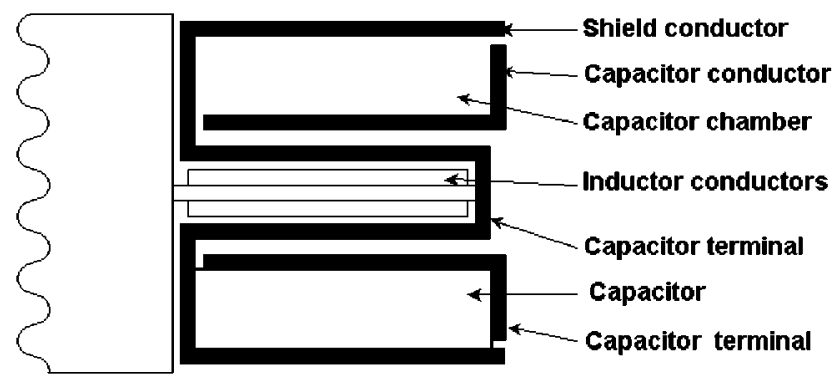

Fig. 10. Section of the proposed shield for the LPF structure.

The section is of the inductor windings outside the core where the capacitors are to be placed.

1) Magnetic Field Distribution: Before the LPF structure is investigated, a single inductor is considered. The magnetic field density $\mathbf{B}$ is calculated for the structure.

A section is taken through the B-field distribution and plotted on an axis that measures across the protruding inductor winding. This is plotted in Fig. 9. From the figure, an important observation can be made. The magnetic field is nonzero above and below the winding. This means that any conducting material placed above or below the conductors will be exposed to induced eddy currents that will increase the losses. To reduce the losses as a consequence of the eddy currents, a shield can be used to minimize the interaction between the changing magnetic field and the capacitors.

2) Introduce a Shield: Fig. 10 shows a cross section of a shield that can be used to create a field-free region in which the capacitors can be mounted. The magnetic field distribution for the shield has also been determined and is plotted in Fig. 11. The top chamber of the shield is left empty, while a simplified capacitor structure has been placed in the lower chamber for the purpose of the simulation. Fig. 11 shows the magnetic field concentration on the inner and outer conductors of the shield. In the two chambers, both with and without the capacitor, the magnetic field density has been reduced as compared to Fig. 9. This will allow the capacitors to operate while in close vicinity to the inductor without an excessive increase in the losses.

The losses in the LPF structure will depend on the design and materials used for the construction of the inductor and capacitors. A shield can be used to prevent the capacitors from suffering extensive losses due to the close proximity of the

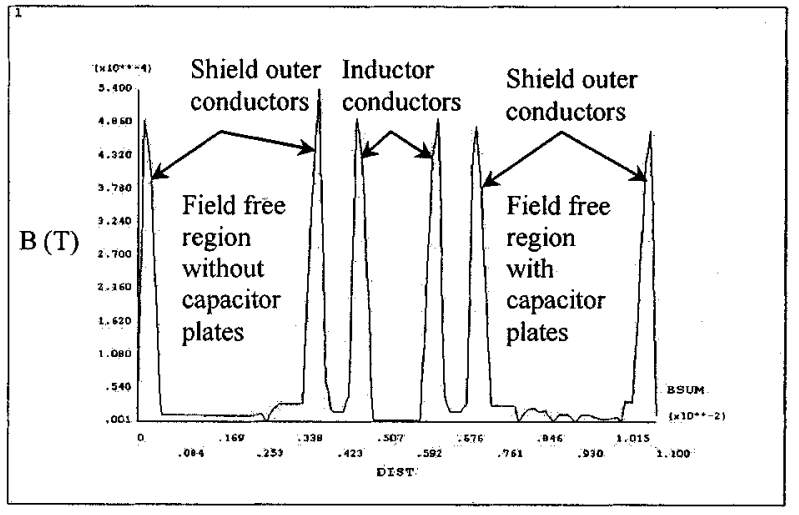

Fig. 11. Magnetic field density distribution along the height of the shield structure.

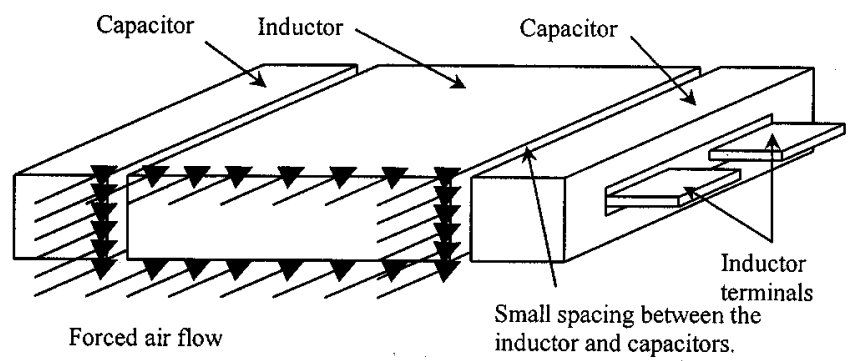

Fig. 12. Cooling the LPF via forced convection.

inductor and capacitors. The shield makes use of eddy currents to establish a region in which the capacitors are protected from the magnetic field of the inductor.

\section{Heat Dissipation}

The LPF consisting of an individual inductor and capacitor has a large total surface area due to the components construction. Any heat generated within the components can be removed via convection or radiation into the surrounding environment. Both these phenomena are proportional to the surface area of the components [7]. However, the proposed LPF structure, assumed to be comparable to the individual inductor and capacitor, will have a smaller surface area exposed due to the dense construction. As a result, less heat can be removed from the structure via convection or radiation. To overcome this problem, there are generally two possible solutions. The first is to use forced convection while the second is to use a heat-sink structure.

1) Forced Convection: The additional heat generated within the structure can be removed with the aid of forced convection. A fast-flowing stream of cooled air can be forced over the surface area of the structure allowing the LPF structure to be efficiently cooled. A second possibility is to include a small gap between the inductor and capacitors of the structure. The stream of air can then be forced between the components as illustrated in Fig. 12.

2) Heat Removal Paths: A second possibility is to use heat conduction to remove the heat from the heat source and then allow the heat to radiate into the environment. One way to achieve this is with the aid of heat sinks as illustrated in Fig. 13. In the diagram, a heat-sink "finger" is included between the inductor and capacitor structures. The heat in these fingers is 


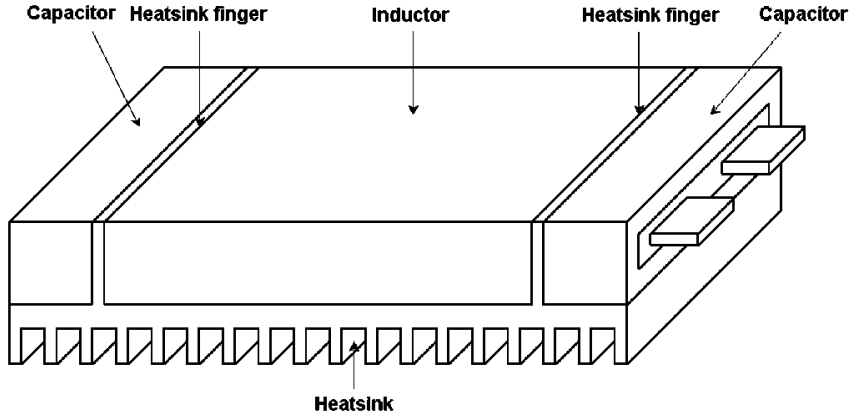

Fig. 13. Removing the heat from the structure with heat sinks.

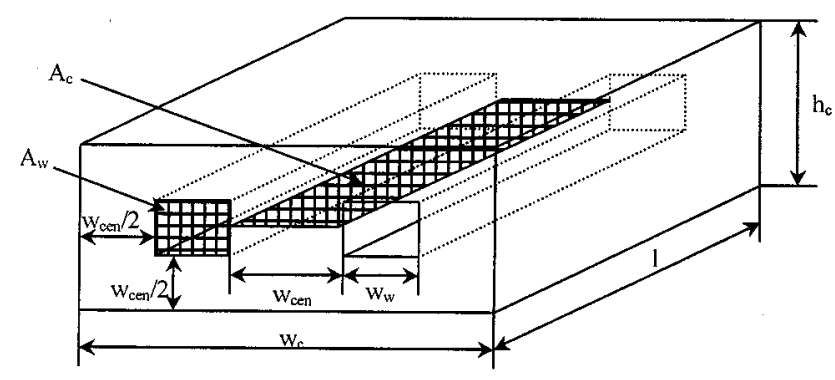

Fig. 14. Double E-core inductor structure (windings are not shown).

then conducted to the main heat sink below the structure as a consequence of conduction. Once the generated heat is in the heat sink, it can be removed via radiation or convection.

\section{VOLUME OPTIMIZATION ANALYSIS}

The volume analysis of the LPF structure is divided into three sections. The first section links the inductors volume to the material properties and operating parameters. The second section links the capacitors volume to the operating parameters and material properties of the capacitor. The results of the two sections are used to find the optimum volume of the LPF structure.

\section{A. Inductor Volume Considerations}

To describe the volume of the inductor in terms of material properties, the following variables are assumed to be known:

- $W_{l}$ the energy to be stored in the inductor;

- $J$ the current density in the conductors;

- $B$ the maximum flux density allowed in the magnetic material.

It is further assumed that the inductor is implemented using a double E-core structure as illustrated in Fig. 14. $A_{w}$ is defined to be the winding window cross-sectional area, while $A_{c}$ is the cross-sectional area of the inductor core.

The energy density in an inductor is

$$
E_{l}=\frac{1}{2} B H \quad \mathrm{~J} / \mathrm{m}^{3}
$$

where $H$ is the magnetic field intensity.

The energy stored within the inductor structure can be rewritten as

$$
W_{l}=\frac{1}{2} \frac{B^{2}}{\mu_{0} \mu_{r}} A_{c} l_{c} \quad \mathrm{~J}
$$

where

$A_{c} \quad$ core cross-sectional area;

$l_{c} \quad$ magnetic length of the core;

$\mu_{0} \mu_{r}$ permeability of the magnetic material.

Equation (2) can be rewritten as

$$
A_{c} l_{c}=\frac{2 \mu_{0} \mu_{r} W_{l}}{B^{2}} \mathrm{~m}^{3} \text {. }
$$

Equation (3) relates the magnetic material properties to the volume required to store the energy in the inductor.

Consider now

$$
N I=\Re \phi \quad \mathrm{A}
$$

where

$N I$ total current in the winding window;

$\phi \quad$ magnetic flux;

$\Re \quad$ reluctance and is defined as [2]

$$
\Re=\frac{l_{c}}{\mu_{0} \mu_{r} A_{c}} \quad \mathrm{H}^{-1} \text {. }
$$

Substituting (4) and (5) into (3) and rearranging, the core volume can be written as

$$
A_{c} l_{c}=\frac{N I \mu_{0} \mu_{r} A_{c}}{B} \mathrm{~m}^{3} .
$$

Equating (3) and (6), the current in the winding window is related to the core volume as

$$
N I=\frac{2 W_{l}}{A_{c} B} \quad \text { A. }
$$

Consider now the winding window. The current in the winding window can be represented by

$$
N I k_{f i l}=A_{w} J \quad \mathrm{~A}
$$

where $k_{f i l}$ is packing factor within the winding window, defined as the ratio of the cross-sectional area of the winding window to the cross-sectional area of the conductors.

Substituting (8) into (7), the relationship between the material properties, operating conditions, and volume is obtained

$$
A_{c} A_{w}=\frac{2 W_{l} k_{f i l}}{B J} \quad \mathrm{~m}^{4} .
$$

Equation (9) illustrates that the product of the winding window area and core cross-sectional area needs to be as small as possible for a given set of excitation variables. It can be seen that, as either the current density or the magnetic field density (or both) is increased, the volume of the magnetic core will decrease, but as the amount of energy stored in the core increases, the volume will increase accordingly. The winding package factor will always increase the volume of the core as a consequence of it representing the unused area of the winding window.

\section{B. Capacitor Volume Considerations}

The relationship between the physical properties of the capacitor and the volume of the capacitor is determined in a simpler way as for the inductor structure. The primary reason for this is 


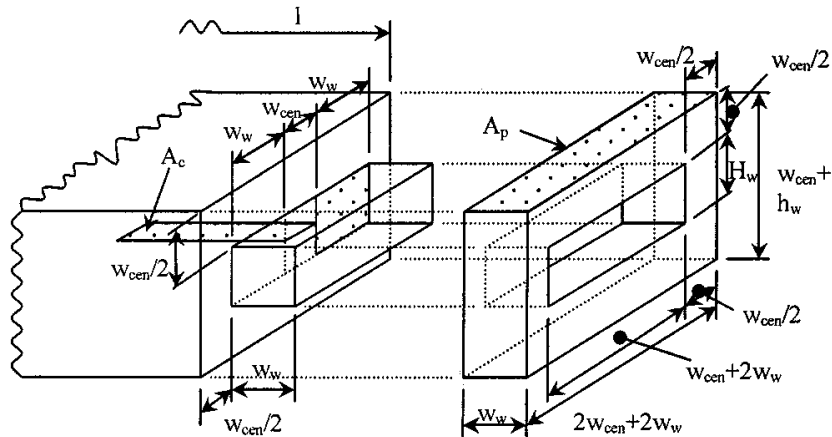

Fig. 15. Section of the inductor and one of the two capacitors. All the defining dimensions are shown in the figure.

that capacitors are mostly available in prepackaged structures, whereas inductors are mostly custom made. It is assumed that the following variables are known: $W_{c}$ the energy to be stored in the capacitor, and $C_{\text {density }}$ the capacitance density.

The energy that is stored in a capacitor in terms of the electrical properties is

$$
W_{c}=\frac{1}{2} C V^{2} \quad \mathrm{~J}
$$

where $C$ is the capacitance of the capacitor, and $V$ is the operating voltage. The capacitor density is defined as

$$
C_{\text {density }}=\frac{C}{C_{\mathrm{vol}}} \quad \mathrm{F} / \mathrm{m}^{3}
$$

where $C_{\mathrm{vol}}$ is the required capacitance volume.

Equation (11) can be rewritten as

$$
C=C_{\mathrm{vol}} C_{\text {density }} \quad \mathrm{F} \text {. }
$$

Substituting (12) into (10), the volume of the capacitor can be determined to be

$$
C_{\mathrm{vol}}=\frac{2 W_{c}}{V^{2} C_{\text {density }}} \quad \mathrm{m}^{3} .
$$

Equation (13) relates the volume of the capacitor to the operation parameters and material properties. The material properties are represented by $C_{\text {density }}$ and will vary for different capacitor technologies and packaging methods used. From (13), it is evident that the volume of the capacitor will increase as the energy to be stored increases. Interestingly, the volume of the capacitor will decrease as the operating voltage increases. It must, however, not exceed the maximum breakthrough voltage of the dielectric.

\section{LPF Volume Considerations}

The inductor and capacitors must now be combined into a single structure in such a way that the volume requirements are met for both devices. One-half of the LPF structure is illustrated in Fig. 15. The structure is defined in terms of four variables: the winding window width $w_{w}$, the height of the winding window $h_{w}$, the width of the center leg of the core $w_{\text {cen }}$, and the length of the core, $l$. In terms of these four variables, the volume of the LPF is given by

\begin{tabular}{|c|c|c|}
\hline Parameter & Symbol & Value \\
\hline Input Voltage & $V_{\mathrm{in}}$ & $42 \mathrm{~V}$ \\
\hline Output voltage & $V_{\mathrm{sex}}$ & $14 \mathrm{~V}$ \\
\hline Converter power & $\mathbf{P}$ & $100 \mathrm{~W}$ \\
\hline Load current & $\mathrm{I}_{\mathrm{igza}}$ & $7.14 \mathrm{~A}$ \\
\hline Operating Frequency & $F_{\mathrm{g} \text { vitch }}$ & $170 \mathrm{kHz}$ \\
\hline Inductance & $\mathrm{L}$ & $2.2 \mu \mathrm{H}$ \\
\hline Capacitance & $\mathrm{C}$ & $5.2 \mathrm{uF}$ \\
\hline Current Density & $\mathrm{J}$ & $2 \mathrm{~A} \mathrm{~mm}^{2}$ \\
\hline Inductor Flux Density & $B$ & $30 \mathrm{mT}$ \\
\hline Copper Package Factor & $\mathrm{k}_{\text {zit }}$ & 3.4 \\
\hline Inductor Energy & $w_{1}$ & $56 \mathrm{uJ}$ \\
\hline Capacitor Voltage & $\mathrm{V}$ & 14 \\
\hline Capacitance Density & $C_{\text {density }}$ & $1 \mathrm{HF} / \mathrm{cm}^{3}$ \\
\hline Capacitor Energy & $W_{8}$ & $510 \mathrm{uJ}$ \\
\hline
\end{tabular}

$$
L P F_{\mathrm{vol}}=C_{\mathrm{vol}}+L_{\mathrm{vol}}
$$

TABLE I

CAse Study Converter Operating Parameters

$$
=\left(l+2 w_{w}\right)\left(2 w_{\text {cen }}+2 w_{w}\right)\left(w_{\text {cen }}+h_{w}\right) \mathrm{m}^{3}
$$

where $L P F_{\mathrm{vol}}$ is the volume of the LPF structure, and $L_{\mathrm{vol}}$ is the inductor volume. To find the volume and, thus, the dimensions of the LPF structure, the four variables mentioned above must be solved.

There are four dimensional variables in (14) and, thus, four equations are required. The first of the four equations is derived from the volume of the capacitor. The capacitor volume in terms of the four variables is

$$
C_{\mathrm{vol}}=2 w_{w} w_{\mathrm{cen}}\left(2 w_{\mathrm{cen}}+2 w_{w}+h_{w}\right) \quad \mathrm{m}^{3} .
$$

The second and third equations are obtained by considering the aspect ratios of the cross-sectional area of the core and the area of capacitor plates. The aspect ratio of the core is given by the ratio of the width of the center leg of the core to its length and is dimensionless

$$
k_{c}=\frac{w_{\mathrm{cen}}}{l} .
$$

The aspect ratio of the capacitor, also dimensionless, is defined as

$$
k_{p}=\frac{2 w_{w}+2 w_{\mathrm{cen}}}{w_{w}} .
$$

The fourth equation is taken from (9), repeated below

$$
A_{c} A_{w}=\frac{2 W_{l} k_{f i l}}{B J} \mathrm{~m}^{4} .
$$

It is not possible to solve for the four variables directly because the equations form an inexplicit system and, thus, need to be solved numerically. To illustrate, a case study is considered.

\section{CASe Study}

The case study is a buck converter chopping $42 \mathrm{~V}$ down to $14 \mathrm{~V}$. The remainder of the converter parameters are listed in Table I. The converter schematic is drawn in Fig. 16. 


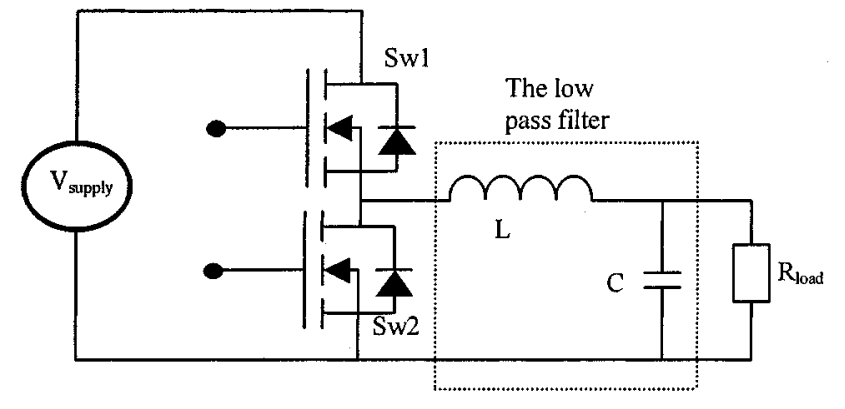

Fig. 16. Buck converter case study.

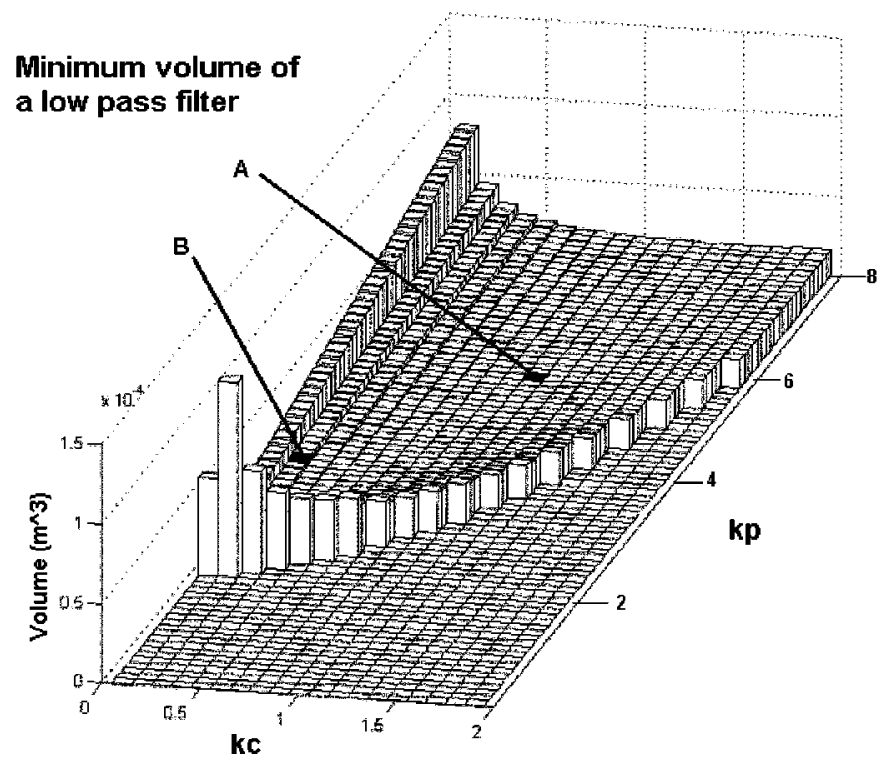

Fig. 17. Volume as a function of $k c$ and $k p$.

\section{A. Volume Optimization}

Using Table I, (13) and (18) can be solved. The results are, respectively,

and

$$
C_{\mathrm{vol}}=\frac{2 W_{c}}{V^{2} C_{\text {density }}}=4.896^{*} 10^{-6} \mathrm{~m}^{3}
$$

$$
A_{c} A_{w}=\frac{2 W_{l} k_{f i l}}{B J}=7.14^{*} 10^{-9} \quad \mathrm{~m}^{4} .
$$

Equations (19) and (20), together with a Matlab program, are used to solve the system and determine the volume of the structure as a function of $k_{c}$ and $k_{p}$. The results of this analysis are plotted in Figs. 17 and 18. Fig. 17 shows the volume of the LPF structure for a range of core and capacitor aspect ratios. The block labeled "A" represents the combination of core and capacitor aspect ratios that produces the lowest volume structure for the case study. The block labeled "B" will be discussed at a later stage. Fig. 18 shows the energy density of the same range of aspect ratios. From the two figures, the trend in the volume relative to the aspect ratios is clear.

The block labeled "A" is the lowest volume point in Fig. 17. As the aspect ratios move away from this trough, the volume increases.

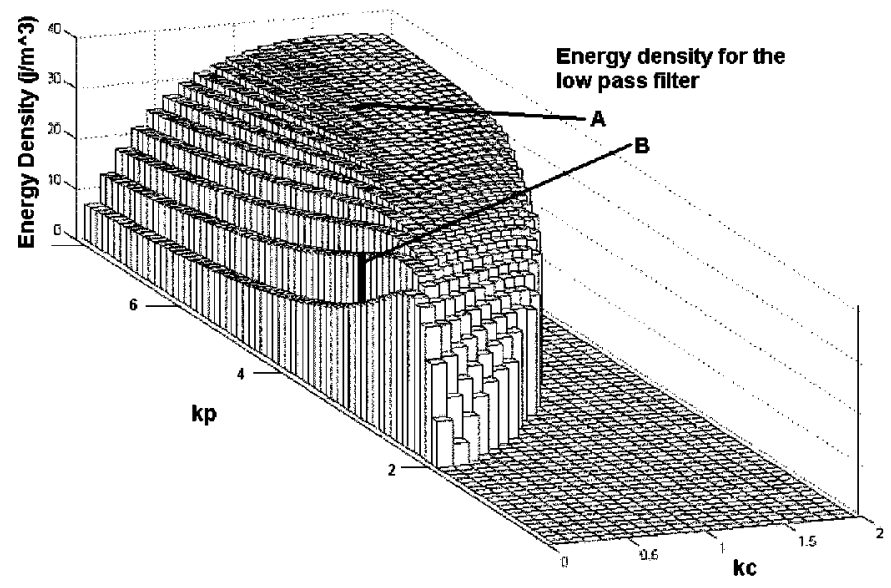

Fig. 18. Energy density as a function of $k c$ and $k p$.

TABLE II

DIMENSIONS FOR THE IDEAL LOW-PASS FILTER STRUCTURE

\begin{tabular}{c|c|c}
\hline Dimension & Symbol & Length [mm] \\
\hline Winding Window Width & $\mathrm{W}_{\mathrm{w}}$ & 6.7 \\
\hline Winding Window Height & $\mathrm{H}_{\mathrm{w}}$ & 11.3 \\
\hline Core Center Leg Width & $\mathrm{W}_{\mathrm{z}}$ & 8.7 \\
\hline Core Length & 1 & 10.9 \\
\hline
\end{tabular}

There is a sudden drop to zero volume for many combinations of aspect ratios. These are unrealizable combinations, resulting in negative dimensions. In Fig. 18, the highest energy density is marked with the block labeled "A." From this point in Fig. 18, the energy density decreases as the aspect ratios move away from this point.

\section{B. Ideal Structure Shape}

From the Matlab program, the dimensions of the structure were determined, and they summarized in Table II. These dimensions represent the LPF that has the lowest possible volume for the given operating conditions. From the dimensions, it can be seen that the ideal structure shape is not planar in nature, but instead tends toward a structure with narrow, tall winding windows. The core itself tends toward a core with a square center leg.

\section{Constructed Filter}

The filter that was constructed to evaluate the LPF structure was built in a planar fashion instead of the fully volume-optimized filter. In Figs. 17 and 18, the blocks labeled "B" represent the filter structure that was implemented. The difference in the shapes of the structures is large, however, the planar structure has a volume that is only $25 \%$ more than that of the optimized LPF. The dimensions of the implemented LPF structure, as well as the volume and energy density, are listed in Table III. When points A and B are compared in Figs. 17 and 18, it can be observed that there are many possible structures that will produce a structure with a volume close to that of the optimum structure. The shape of the LPF structure will change significantly over the range of acceptable volumes allowing the physical operating environment (e.g., structure profile) to have some influence over the finalized structure shape. 
TABLE III

DIMENSIONS OF THE CONSTRUCTED LOW-PASS FILTER

\begin{tabular}{c|c|c}
\hline Dimension & Symbol & Length [mm] \\
\hline Winding Window Width & $\mathrm{W}_{\mathrm{m}}$ & 8.5 \\
\hline Winding Window Height & $\mathrm{H}_{\mathrm{rc}}$ & 3 \\
\hline Core Center Leg Width & $\mathrm{W}_{\mathrm{con}}$ & 8 \\
\hline Core Length & 1 & 35 \\
\hline Structure Volume & & $18800 \mathrm{~mm}^{3}$ \\
\hline Energy Density & & $28.66 \mathrm{~J}^{3}$ \\
\hline
\end{tabular}

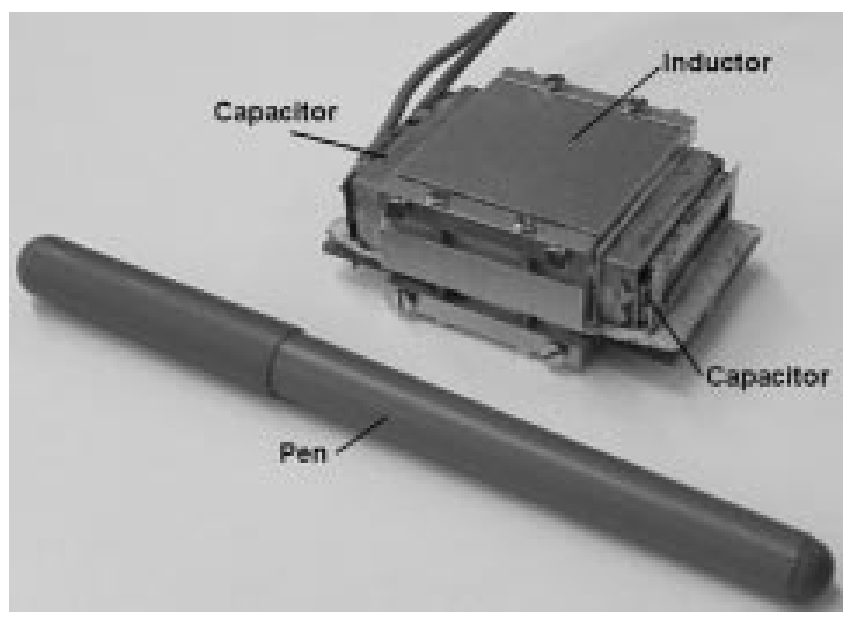

Fig. 19. Photograph of the low-pass filter structure.

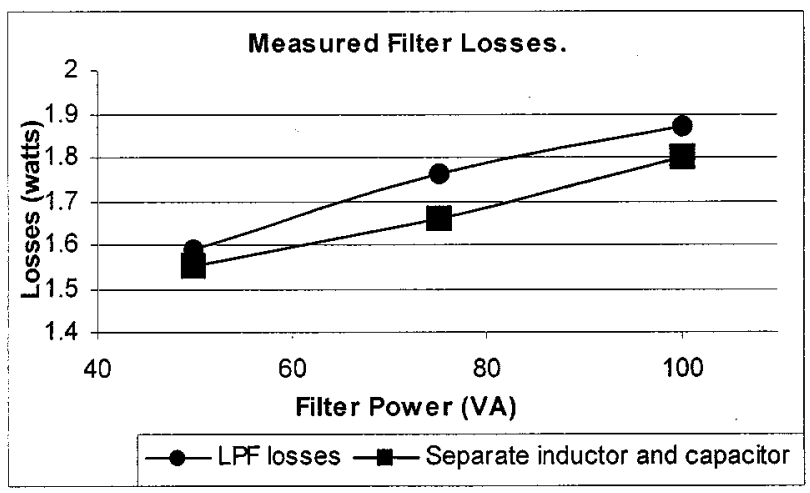

Fig. 20. LPF losses as a function of output power.

The interconnections in the constructed filter were implemented with the microstrip line method proposed earlier. This method was selected for the relative ease that the interconnections can be implemented. A heat-sink structure was also used in conjunction with the LPF structure. The purpose of the heat sink is twofold. The first is to provide a low thermal resistance to the surrounding environment to ensure that the structure does not suffer damages due to overheating. The second is mechanical rigidity. The inductor is implemented with a distributed air-gap structure and the heat sink is used to hold the structure together.

\section{Measured Results}

Fig. 19 shows a photograph of the implemented LPF structure. Fig. 20 shows the measured losses as a function of excitation power for two conditions. The first is the loss measurement
Loss distribution at 100VA excitaion

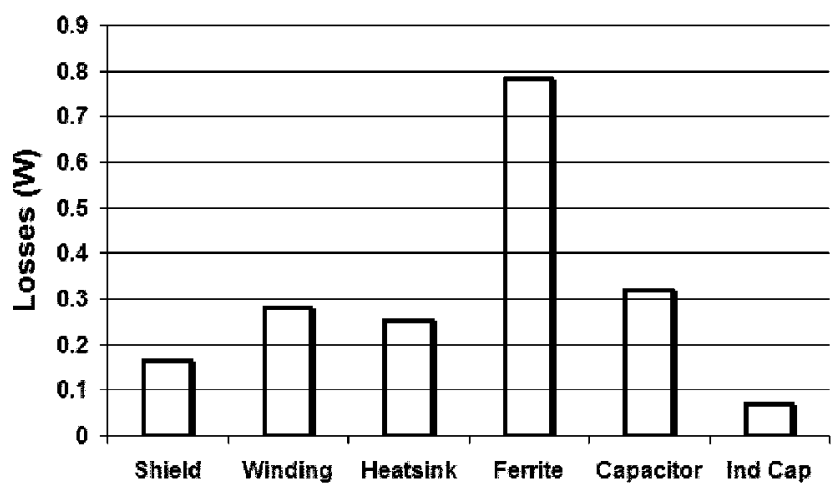

Fig. 21. LPF loss distribution.

for the complete LPF with the implemented shield. The second is for the complete LPF structure but with the capacitor and inductor physically separated from each other. There is a constant difference in the measured power loss which is accounted for by the additional losses induced in the shield and capacitors. Fig. 21 shows the loss distribution for the LPF structure. The chart is a combination of the measured and theoretical loss distribution. The $17 \%$ capacitor loss is the measured losses in the capacitors under normal operation without the interaction between the inductor and capacitors. On combining the two components with the shield, the capacitor losses increase by $4 \%$ and is referred to as the induced capacitor losses ("Ind Cap"). Without the shield, the induced losses in the capacitors would be large enough to destroy the capacitors. The small increase in the capacitor losses is an indication of the effectiveness of the shield.

\section{CONCLUSION}

In this paper, it was shown that, if the construction method for an LPF is considered, it is possible to achieve a structure that utilizes the available volume more efficiently than the standard LPF construction. This is achieved by building the filter construction in an unconventional manner. Several practical issues concerning the construction of such a filter structure were identified and discussed. These included interconnections, heat removal, and a shield to protect the capacitors. A volume optimization analysis was performed to help determine the optimum dimensions for such a filter structure. An LPF was then built using the issues that were previously identified. The structure was successfully implemented in a $100-\mathrm{W}$ dc/dc converter. The proposed LPF structure has an energy density of approximately $28 \mathrm{~J} / \mathrm{m}^{3}$ as compared to approximately $18-22 \mathrm{~J} / \mathrm{m}^{3}$ for the conventional LPF structures for the same parameters, showing an improvement of approximately $25 \%$.

\section{REFERENCES}

[1] J. D. Van Wyk and F. C. Lee, "Power electronic technology at the dawn of the new millennium-status and future," in Proc. IEEE PESC'99, vol. 1, 1999, pp. 3-12.

[2] J. D. Kraus and D. A. Fleisch, Electromagnetics With Applications. New York: McGraw-Hill, ch. 2 and 7. 
[3] J. T. Strydom, J. D. van Wyk, and J. A. Ferreira, "Electromagnetic limits of planar integrated resonant/transformer structures for power electronic applications," in Proc. Int. Workshop Integrated Power Packaging (IWIPP 2000), 2000, pp. 97-101.

[4] J. A. Bently, M. Hudis, R. M. Kerrigan, and F. W. MacDougall, "High frequency performance of power film capacitors," in Conf. Rec. IEEE-IAS Annu. Meeting, vol. 3, 1995, pp. 2521-2528.

[5] R. Reeves, "Choke-capacitor hybrids as a fluorescent lamp ballast," Proc. Inst. Elect. Eng., vol. 122, no. 10, pp. 1151-1152, 1975.

[6] A. B. Lostetter, F. Barlow, A. Elshabini, K. Olejniczak, and S. Ang, "Polymer thick film and Flex technologies for low cost power electronics packaging," in Proc. Int. Workshop Integrated Power Packaging (IWIPP 2000), 2000, pp. 33-40.

[7] J. Lammeraner, Eddy Currents. Prague, Czechoslovakia: SNTL, 1964, ch. 1 and 2 .

[8] N. Mohan, T. M. Undeland, and W. P. Robbins, Power Electronics. Converters, Applications and Design, 2 ed. New York: Wiley, 1995.

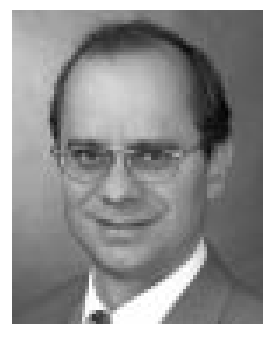

Jan Abraham Ferreira was born in Pretoria, South Africa. He received the B.Sc.Eng., M.Sc.Eng., and $\mathrm{Ph} . \mathrm{D}$. degrees in electrical engineering from Rand Afrikaans University, Johannesburg, South Africa, in 1980, 1982, and 1988, respectively.

He was with ESD (Pty) Ltd. from 1982 to 1985. From 1986 to 1997, he was with the Faculty of Engineering, Rand Afrikaans University, where he held the Carl and Emily Fuchs Chair of Power Electronics. Since 1998, he has been a Professor in the ITS Faculty, Delft University of Technology, Delft, The Netherlands. He has coauthored more than 150 publications on the topic of power electronics. He is a member of the Executive Committee of the EPE Society.

Prof. Ferreira is the TRAnSACtions Review Chairman of the Power Electronics Devices and Components Committee of the IEEE Industry Applications Society (IAS). He is the Founding Chairman of the IEEE Joint IAS/Power Electronics Society Benelux Chapter. He is also a member of the IEEE Power Engineering Society AdCom.

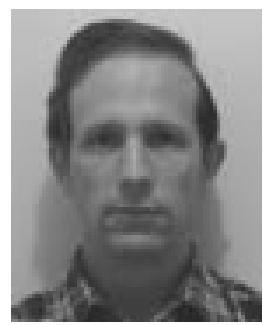

Ivan W. Hofsajer (M'93) was born in Johannesburg, South Africa. He received the B.Ing, M.Ing, and D.Ing degrees in electrical engineering from Rand Afrikaans University, Johannesburg, South Africa, in 1991, 1993, and 1998, respectively.

He was with the South African Atomic Energy Corporation, working in the field of electromagnetic interference. He is currently a Senior Lecturer in the Department on Electrical and Electronic Engineering, Rand Afrikaans University. His interests include power electronics and electromagnetics.

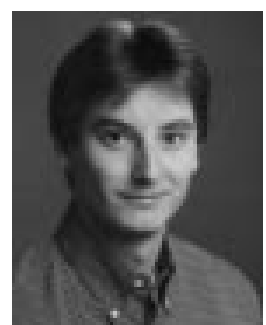

Norbert Seliger received the Ph.D. degree in electrical engineering from the Institute for Solid State Electronics, Technical University of Vienna, Vienna, Austria, in 1998.

Since 1998, he has been with Corporate Technology, Siemens AG, Munich, Germany, where his research activities involve compact power electronics and high-temperature electronics. 